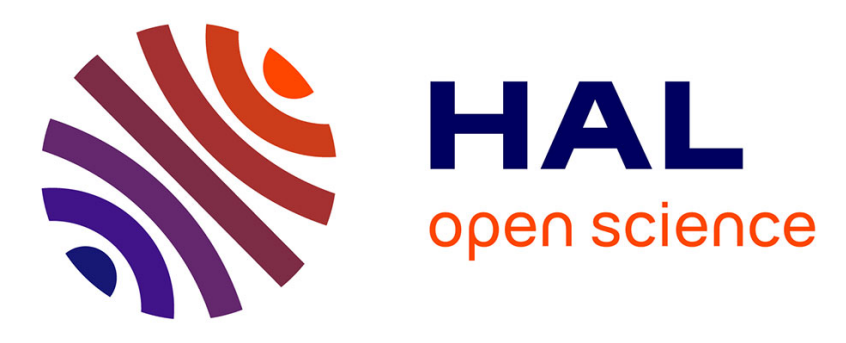

\title{
Brunel-Dominated Proton Acceleration with a Few-Cycle Laser Pulse
}

M. Veltcheva, A. Borot, C. Thaury, A. Malvache, E. Lefebvre, A. Flacco, R. Lopez-Martens, V. Malka

\section{- To cite this version:}

M. Veltcheva, A. Borot, C. Thaury, A. Malvache, E. Lefebvre, et al.. Brunel-Dominated Proton Acceleration with a Few-Cycle Laser Pulse. Physical Review Letters, 2012, 108 (7), pp.075004. 10.1103/PhysRevLett.108.075004 . hal-01166881

\section{HAL Id: hal-01166881 https://hal.science/hal-01166881}

Submitted on 23 Jun 2015

HAL is a multi-disciplinary open access archive for the deposit and dissemination of scientific research documents, whether they are published or not. The documents may come from teaching and research institutions in France or abroad, or from public or private research centers.
L'archive ouverte pluridisciplinaire HAL, est destinée au dépôt et à la diffusion de documents scientifiques de niveau recherche, publiés ou non, émanant des établissements d'enseignement et de recherche français ou étrangers, des laboratoires publics ou privés. 


\title{
Brunel-Dominated Proton Acceleration with a Few-Cycle Laser Pulse
}

\author{
M. Veltcheva, ${ }^{1,2}$ A. Borot, ${ }^{1}$ C. Thaury, ${ }^{1}$ A. Malvache, ${ }^{1}$ E. Lefebvre, ${ }^{3}$ A. Flacco, ${ }^{1}$ R. Lopez-Martens, ${ }^{1}$ and V. Malka ${ }^{1}$ \\ ${ }^{1}$ Laboratoire d'Optique Appliquée, ENSTA-PARISTECH, CNRS, Ecole Polytechnique, UMR 7639, 91761 Palaiseau, France \\ ${ }^{2}$ Dipartimento di Fisica “G. Occhialini," Universitá degli Studi Milano-Bicocca, piazza della Scienza 3, 20126 Milan, Italy \\ ${ }^{3}$ CEA, DAM, DIF, 91297 Arpajon, France
}

(Received 12 May 2011; published 17 February 2012)

\begin{abstract}
Experimental measurements of backward accelerated protons are presented. The beam is produced when an ultrashort ( $5 \mathrm{fs}$ ) laser pulse, delivered by a $\mathrm{kHz}$ laser system, with a high temporal contrast $\left(10^{8}\right)$, interacts with a thick solid target. Under these conditions, proton cutoff energy dependence with laser parameters, such as pulse energy, polarization (from $p$ to $s$ ), and pulse duration (from 5 to 500 fs), is studied. Theoretical model and two-dimensional particle-in-cell simulations, in good agreement with a large set of experimental results, indicate that proton acceleration is directly driven by Brunel electrons, in contrast to conventional target normal sheath acceleration that relies on electron thermal pressure.
\end{abstract}

About a decade ago, several experiments demonstrated the possibility of accelerating ions through the interaction of an ultraintense, subpicosecond laser pulse with a solid target $[1,2]$. Since then, our knowledge about this phenomenon has increased through a number of studies relating the ion cutoff energy and spectral features to various laser and target parameters [3-8]. Laser-driven ion sources are particularly interesting in medicine for hadrontherapy $[9,10]$ and in science for plasma radiography [11], heating particles for fast ignition [12], and warm dense matter studies $[13,14]$. The properties of the ion beam also provide useful information in situ about the interaction conditions, including laser focusing and temporal contrast $[15,16]$.

When an ultraintense pulse interacts with a solid surface, a plasma is created and part of the laser energy is transferred to the plasma electrons. Depending on the laser intensity, several regimes can be distinguished. For medium intensities $\left(10^{16} \mathrm{~W} / \mathrm{cm}^{2} \lesssim I_{\text {laser }} \lesssim 10^{18} \mathrm{~W} / \mathrm{cm}^{2}\right)$, the electron velocity is $v_{e} \ll c$ and the dominant force acting on the electrons is the laser electric field, setting an oscillatory motion in the direction of laser polarization. For relativistic laser intensities $\left(I_{\text {laser }}>10^{18} \mathrm{~W} / \mathrm{cm}^{2}\right)$, the magnetic component of the Lorentz force becomes important and electrons are pushed in the forward direction by the $\mathbf{j} \times \mathbf{B}$ force set by the laser [5,17]. Independently of the details of the acceleration regime, electron motion creates a strong charge separation and sets up $\mathrm{a} \approx \mathrm{TV} / \mathrm{m}$ electrostatic field at the target-vacuum interface. This field accelerates ions out of the target. The scenario is commonly called target normal sheath acceleration (TNSA) and is the most accredited mechanism for ion acceleration $[18,19]$.

We present here the first observation of ion acceleration by high-contrast, few-cycle laser pulses at $1 \mathrm{kHz}$ repetition rate on a thick solid target. Differently from classical TNSA, in this condition, the acceleration is found to be directly driven by Brunel electrons, dragged in and out of the plasma by the laser electric field [20] and not by the electron thermal pressure. As a result, the acceleration takes place only within the laser pulse duration, in contrast with [4], where the Brunel electrons are trapped in the space-charge field at the target edges, leading to classical TNSA acceleration on a $p s$ time scale. The new accelerating mechanism is investigated through a systematic study of the dependence of proton cutoff energy on laser pulse energy, polarization, and duration. The results are supported by $2 \mathrm{D}$ particle-in-cell (PIC) simulations and an analytical model that reveals the underlying physics of the interaction.

The experiment was performed with the Salle Noire Ti: Sa laser facility $(\lambda=800 \mathrm{~nm})$ at the Laboratoire d'Optique Appliquée, which delivers up to $1 \mathrm{~mJ}$ in $5 \mathrm{fs}$ at a $1 \mathrm{kHz}$ repetition rate [21]. The few-cycle pulse duration is achieved via spectral broadening of $25 \mathrm{fs}$ pulses in a helium-filled hollow-core fiber followed by compression with ultrabroadband chirped mirrors. The laser pulses have a measured temporal contrast ratio of $10^{8}$ in the fewpicosecond range. Under vacuum, the $p$-polarized pulses are tightly focused with an $f / 1.7$ off-axis parabola (OAP) (1.7 $\mu \mathrm{m}$ FWHM spot size) at oblique incidence $\left(57^{\circ}\right)$ onto the surface of a continuously refreshed, optically polished, thick glass target, which enables us to fully exploit the $\mathrm{kHz}$ laser pulse. To ensure identical interaction conditions, the moving target surface is monitored and stabilized using Mach-Zehnder interferometry. This way, the residual target surface motion is below $2 \mu \mathrm{m}$, corresponding to a fraction of the Rayleigh length of the beam. Identical experimental conditions are used to observe high-order harmonic generation from the target [22].

The ion beam, generated mostly from the contaminant deposition on the target surface, is spatially filtered by a $300 \mu \mathrm{m}$ pinhole and observed along the target normal with a Thomson parabola system coupled to a microchannel plate and a phosphor screen detector. Traces are recorded 


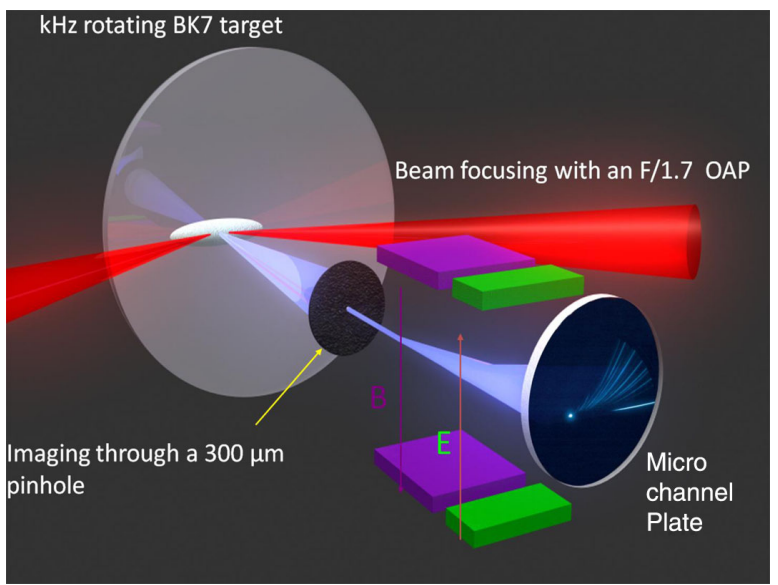

FIG. 1 (color online). Scheme of the experimental setup.

using a 12-bit CCD camera, and each image is integrated over 30 consecutive laser shots. Ion spectra are dispersed in energy and the $q / m$ ratio, respectively, by the magnetic and electric fields in the Thomson parabola. This detection configuration allows us to identify the different ion species and to calculate the beam energy spectrum (Fig. 1).

The first behavior to be analyzed is the dependence on pulse energy. A $\lambda / 2$ wave plate and a polarizer are mounted in the beam path. The pulse energy is varied by rotating the wave plate and measured before each shot sequence. Figure 2 shows that the proton energy scales as $E_{\max } \propto\left(I_{\text {laser }} \lambda^{2}\right)^{\beta}$ for two different pulse durations, namely, with $\beta=0.57$ for $\tau_{l}=5$ fs and $\beta=0.62$ for $\tau_{l}=26$ fs. A maximum proton energy of $36 \pm 3.6 \mathrm{keV}$ is obtained for $5 \mathrm{fs}$ pulses and $88 \pm 14 \mathrm{keV}$ for $26 \mathrm{fs}$ pulses.

The high temporal contrast of the Salle Noire laser warrants very steep plasma density gradients at the target surface. This condition and the oblique incidence of the beam imply that the Brunel effect [20] should be the dominant electron acceleration mechanism. To support this hypothesis, the laser polarization is varied from $p$ to $s$ while the pulse energy is kept constant. Related proton cutoff energies are shown in the inset of Fig. 2 and are well fitted by $E_{\max } \propto\left[\cos (2 \theta)^{2}\right]^{0.5}$, where $\theta$ is the angle between the optical axis of the wave plate and the direction of laser polarization. This proves that the acceleration is mainly driven by the $p$ component of the laser electric field, confirming that the Brunel effect plays a key role in the acceleration [4,23]. The energy drop, when going from $p$ to $s$ polarization, also confirms the high temporal contrast of the laser.

To complete the study of the dependence of proton cutoff energy on different laser parameters, the influence of the pulse length was also analyzed. To vary the laser pulse duration over more than 1 order of magnitude, the gas in the hollow fiber was removed and chirped mirrors optimized for $25 \mathrm{fs}$ pulse compression were used. The laser pulse can then be stretched from 25 to 500 fs using an

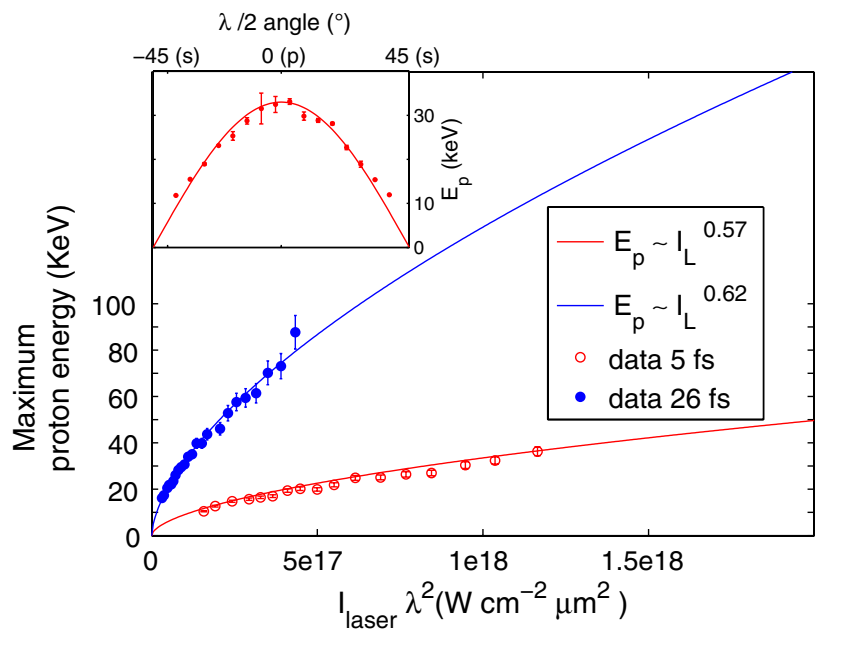

FIG. 2 (color online). Scaling of maximum proton energy with laser intensity on target for two different pulse durations. The maximum laser intensity is $I=1.8 \times 10^{18} \mathrm{~W} / \mathrm{cm}^{2}$ for $\tau_{l}=5 \mathrm{fs}$ (open red circles) and $I=6 \times 10^{17} \mathrm{~W} / \mathrm{cm}^{2}$ for $\tau_{l}=26 \mathrm{fs}$ (filled blue circles). In the inset is the variation in proton energy with laser polarization. The minimum energy detection is limited by the instrument. Continuous lines are fits of the experimental data.

acousto-optic programmable dispersive filter (Dazzler) inside the laser system to change the second-order spectral phase of the pulses. In this study, the pulse energy was kept constant. Figure 3 shows a steep increase in maximum proton energy between $\tau_{l}=25 \mathrm{fs}$ and $\tau_{l}=40 \mathrm{fs}$, then a plateau until $200 \mathrm{fs}$, followed by a slow decrease for longer pulses. The trend indicates that, for the parameters we consider, the highest proton energy is not reached at the maximum laser intensity. In other words, for a constant energy on target, there is an optimal pulse length range for ion acceleration.

The dependence on laser intensity and pulse length was studied through the development of an analytical model

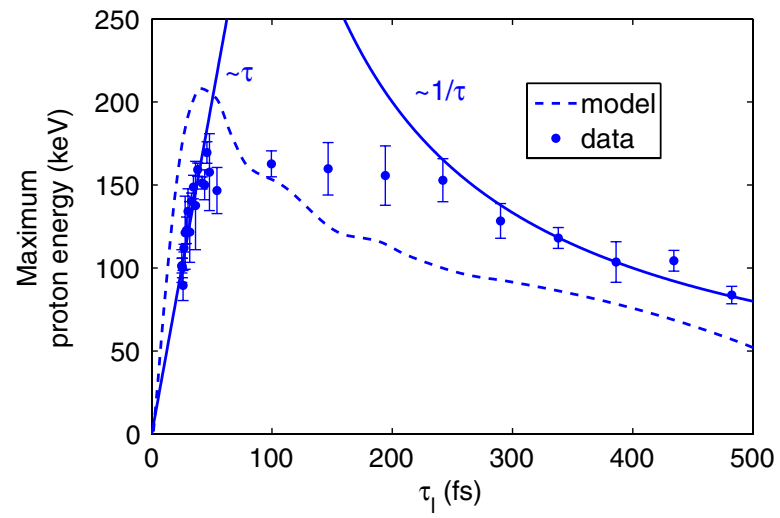

FIG. 3 (color online). Maximum proton energy scaling as a function of laser pulse duration with constant pulse energy. The laser intensity increases from $I \simeq 3 \times 10^{16} \mathrm{~W} / \mathrm{cm}^{2}$ for $\tau_{l}=$ 500 fs up to $I \simeq 6 \times 10^{17} \mathrm{~W} / \mathrm{cm}^{2}$ for $\tau_{l}=25 \mathrm{fs}$. 
describing the dynamics of acceleration in our conditions. During the interaction of an intense laser with a thick solid target, for very steep density gradients, the electrons are dragged out of the surface by the laser field, through the Brunel mechanism [20,24]. Then, electrons are sent back into the plasma, as shown in Fig. 4(a). Once they pass the critical surface, they are no longer under the influence of the laser electric field. Most of the electron energy therefore flows into the bulk of the target, and no significant heating occurs in the plasma. As a result, electron thermal pressure hardly contributes to the ion acceleration, in contrast with "classical" TNSA [18]. The accelerating process can thus be modeled by simply treating the electrostatic field around the plasma surface during the laser-target interaction.

The principle of the model is developed as follows. We assume that $(\boldsymbol{x}, \boldsymbol{y}, \boldsymbol{z})$ is a standard base, with $\boldsymbol{x}$ perpendicular to the plasma surface and $z$ perpendicular to the incidence plane. Electrons' trajectories are obtained from the relativistic equation of motion, taking into account both the $p$-polarized laser field and the electric field due to the electron motion $E_{p}$. The plasma field $E_{p}$ is calculated from Poisson's equation using the self-consistent ion density profile. The plasma is considered to be cold and neutral at the beginning of each optical cycle, and the electron orbits are assumed not to cross in the vacuum $(x<0)$. The field felt by an electron at $x$ is $E_{p}(x)=$ $\left(e / \epsilon_{0}\right) \int_{x}^{\infty}\left(n_{e}-Z n_{i}\right) d x^{\prime}$, with $n_{i}$ the ion density and $Z$

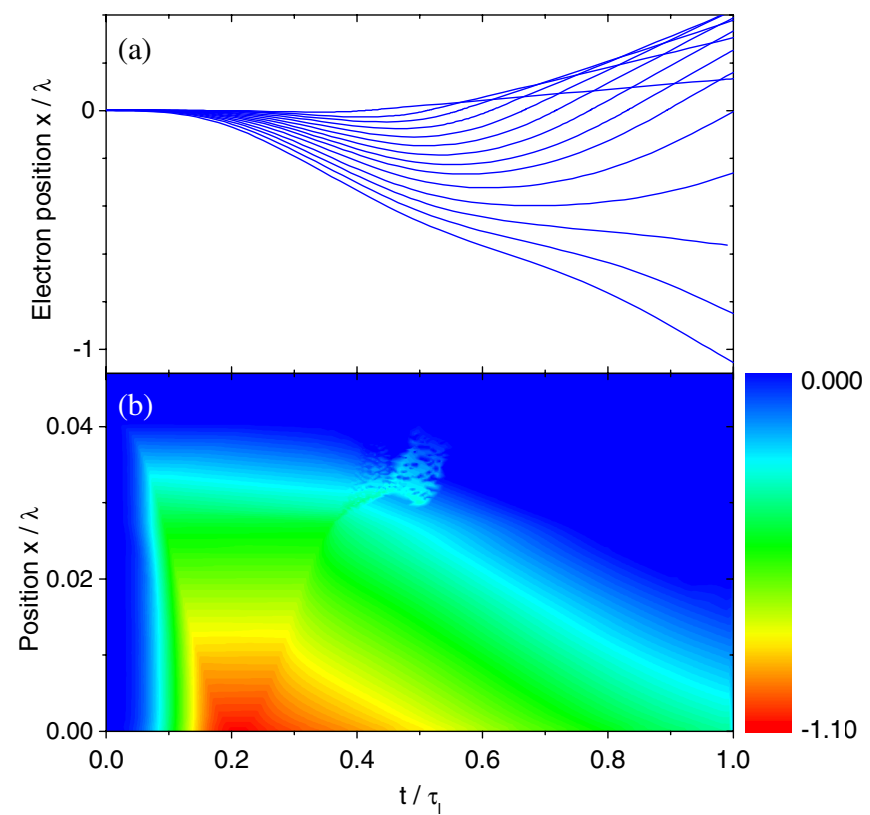

FIG. 4 (color online). (a) Electron trajectories during one laser optical cycle for $\tau_{l}=5$ fs and $a_{0}=0.9$. The plasma occupies the half-space $x \geq 0$, and $t=0$ corresponds to the maximum of the laser pulse envelope. Each trajectory is associated to a slightly different initial position $x_{0}$. (b) Electric field $E_{p}$ in the plasma due to the electron motion. the ion charge. Since electron trajectories do not cross, $\int_{x}^{\infty} n_{e} d x^{\prime}=\int_{x_{0}}^{\infty} n_{e 0} d x^{\prime}=\int_{x_{0}}^{\infty} Z n_{i} d x^{\prime}$, with $x_{0}$ the initial position of the considered electron. It follows that $E_{p}=$ $\left(-\mathrm{Ze} / \epsilon_{0}\right) \int_{x_{0}}^{x} n_{i} d x^{\prime}[24]$.

Although the model is one-dimensional, the oblique incidence is described by making a Lorentz transformation from the laboratory frame to a frame moving parallel to the surface [25]. For the sake of simplicity, the laser absorption is assumed to be constant and equal to $f=0.3$. The equation of motion for electrons in the boosted frame is thus

$$
\begin{aligned}
\frac{d \gamma \boldsymbol{v} / c}{d \omega t}= & -A(t) a_{0}\left([\sin (\omega t-k x)+\eta \sin (\omega t+k x)] \frac{\boldsymbol{v}}{c} \times \boldsymbol{z}\right. \\
& +[\sin (\omega t-k x)-\eta \sin (\omega t+k x)] \boldsymbol{y})-E_{p}^{\prime} \boldsymbol{x},
\end{aligned}
$$

where $A(t)$ is the laser envelope, $\eta=\sqrt{1-f}$ and $E_{p}^{\prime}=$ $e E_{p} / m_{e} c \omega$, and $a_{0}=e E_{L} / m_{e} \omega c$ is the normalized laser vector potential, with $E_{L}$ and $\omega$ as, respectively, the amplitude and the frequency of the laser. Within each laser cycle, Eq. (1) is numerically integrated using the fourthorder Runge-Kutta method. Ions are treated as macroparticles which are moved at the end of each cycle using the field $E_{p}$ resulting from the integration [Fig. 4(b)].

The predictions of the model are now compared with experimental results. The initial density gradient is considered infinitely steep; the electron density is $n_{e 0}=100 n_{c r}$, and the incidence angle $\theta=57^{\circ}$. As shown in Fig. 3, the calculations reproduce well the increase in the ion peak energy $E_{\max }$ with pulse duration for short pulses and the optimum pulse duration and the decrease in $E_{\max }$ for longer pulses. These three features can be explained in the scope of the model.

The amplitude of $E_{p}$ is proportional to $a_{0} m_{e} c \omega / e$ [20,24], with a proportionality factor $\alpha$ that depends mainly on the incidence angle and on the density gradient. In general, $\alpha$ evolves in time since the density gradient changes during the acceleration. However, when the length of the expanding plasma $L$ is smaller than the electron orbit length $x_{\mathrm{osc}}$, the electron dynamics and hence $\alpha$ depend weakly on $L$. In this case, the ion front velocity is $v_{f}(t) / c \approx \alpha a_{0}\left(Z m_{e} / m_{i}\right) \omega \int_{0}^{t} A\left(t^{\prime}\right) d t^{\prime}$, where $m_{i}$ is the ion mass. As a consequence, $E_{\max } \propto a_{0}^{2} \tau_{l}^{2}$ for all $\tau_{l}$, with $L\left(\tau_{l}\right)<x_{\text {osc }}$. In other words, the peak ion energy is proportional to the laser pulse energy for a constant pulse length and proportional to the pulse length for a constant laser energy.

To estimate the domain in which this scaling law is valid in the nonrelativistic limit for an initially steep density gradient, we calculate $L(t)=\int_{0}^{t} v_{f} d t^{\prime}$ and approximate $x_{\text {osc }}$ by its value in a vacuum $x_{\text {osc }} \approx a_{0} c / \omega$ for $a_{0} \lesssim 1$. The approximation is reasonable if the mean electron density in the accelerated plasma is smaller than the critical 


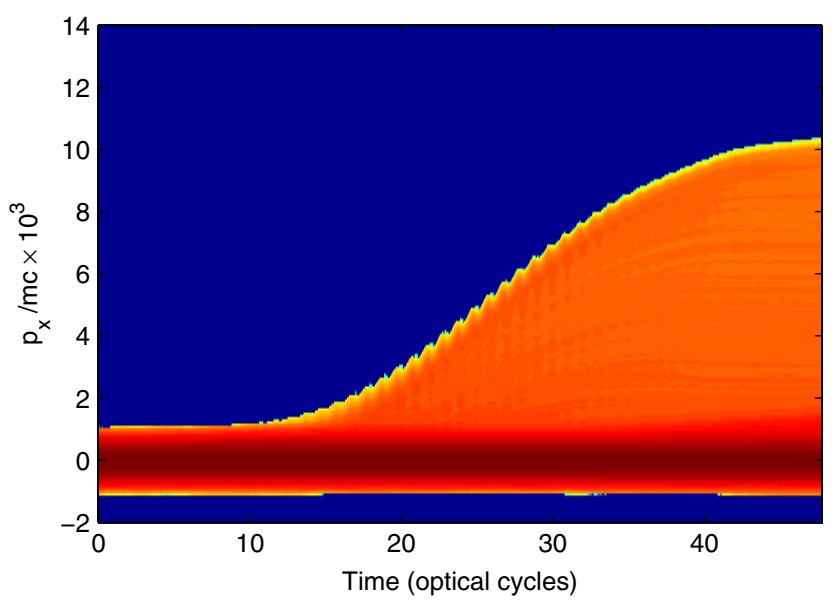

FIG. 5 (color online). Evolution of momentum distribution for $\tau_{l}=25$ fs and $a_{0}=0.4$.

density $n_{c r}$. It follows that, for a square pulse of full length $\tau_{l}$, the condition $L\left(\tau_{l}\right)<x_{\mathrm{osc}}$ is verified only if

$$
\tau_{l} \lesssim \tau_{0}=\frac{1}{\omega} \sqrt{\frac{2}{\alpha} \frac{m_{i}}{Z m_{e}}} .
$$

From the numerical integration of the equation of motion, we evaluate for $\theta=57^{\circ}$ and $f=0.3, \alpha \approx 1$.1. Using this estimate in Eq. (2), we obtain, for $m_{i} / m_{e}=1836$ and $Z=1, \tau_{0} \approx 25 \mathrm{fs}$. The scaling law $E_{\max } \propto a_{0}^{2} t_{\text {end }}^{2}$ is thus valid only for pulse duration $\tau_{l} \lesssim 25$ fs. Accordingly, we observe in Fig. 3 that the maximum proton energy predicted by the model stops increasing linearly with $\tau_{l}$ for $\tau_{l}>25$ fs.

For $\tau_{l} \gg \tau_{0}, x_{\mathrm{osc}} \ll L\left(\tau_{l}\right)$, so the laser drags few electrons beyond the ion front. Consequently, the plasma field $E_{p}$ around the ion front progressively tends to zero and acceleration of the ion front eventually stops at $t_{\text {end }} \propto \tau_{0}$, when almost no electrons are pulled into the vacuum. It follows that, for $\tau_{l}>t_{\text {end }}, E_{\max } \propto a_{0}^{2} t_{\text {end }}^{2}$. At constant laser energy, $a_{0}^{2} \propto 1 / \tau_{l}$ and the scaling law becomes $E_{\max } \propto$ $1 / \tau_{l}$. Figure 3 shows that the asymptotic laws for $\tau_{l} \lesssim$ $\tau_{0}\left(\tau_{l}\right)$ and $\tau_{l} \gg \tau_{0}\left(1 / \tau_{l}\right)$ accurately reproduce the behavior of $E_{\max }\left(\tau_{l}\right)$ in the two extreme cases.

To support the experimental results and validate the model thesis, 2D simulations were performed with the PIC code CALDER [26]. The laser is p-polarized and Gaussian in space and time, with a pulse length of $25 \mathrm{fs}$ (FWHM). It strikes the target at an angle of $45^{\circ}$, with respect to the target normal, and is focused on a spot size of $4 \lambda$ (FWHM), where $\lambda=800 \mathrm{~nm}$ is the laser wavelength. The target is a fully ionized hydrogen plasma with an exponential density gradient scale length of $\lambda / 100$ and of maximum density $n_{e}=200 n_{c r}$. The normalized laser vector potential $a_{0}$ is 0.4 , reproducing the experimental intensity. The simulations are run over a 50 fs time window. In Fig. 5, the evolution of momentum distribution is plotted. The figure shows clearly that protons are accelerated only within the laser pulse duration and that the proton energy increases with the periodicity of the laser field, in agreement with the model.

In conclusion, proton acceleration at a $\mathrm{kHz}$ rate, with a few-cycle, sub-mJ laser system, was presented. A systematic parameter study shows the dependence of proton cutoff energy on laser intensity and polarization. Trends with varying laser pulse duration indicate that there is an optimum $\tau_{l}$ (around 40-50 fs) that maximizes the peak energy of the proton beam in this regime. Acceleration with steep density gradients is found to be dominated by a Brunel-like mechanism without significant contribution from electron thermal pressure. In the future, with the development of $\mathrm{kHz}$ laser systems with higher pulse energy, this kind of source could have an interest for medical applications, such as radioisotope production for positron emission tomography (PET), for which high repetition rates are required [27,28].

We acknowledge the support of the European Research Council for funding the PARIS ERC project (Contract No. 226424). The PIC simulations were carried out on the CCRT and CINES computers thanks to GENCI allocations, Grants No. 2011-056304 and No. 2011-056057, and on the CURIE computer made available by PRACE (SOULAC project).

[1] R. A. Snavely et al., Phys. Rev. Lett. 85, 2945 (2000).

[2] E. L. Clark et al., Phys. Rev. Lett. 84, 670 (2000).

[3] F. Lindau et al., Phys. Rev. Lett. 95, 175002 (2005).

[4] T. Ceccotti et al., Phys. Rev. Lett. 99, 185002 (2007).

[5] J. Fuchs et al., Nature Phys. 2, 48 (2005).

[6] L. Robson et al., Nature Phys. 3, 58 (2006).

[7] B. Hou et al., Appl. Phys. Lett. 95, 101503 (2009).

[8] J. Schreiber et al., Phys. Rev. Lett. 97, 045005 (2006).

[9] S. V. Bulanov et al., Phys. Lett. A 299, 240 (2002).

[10] V. Malka et al., Med. Phys. 31, 1587 (2004).

[11] M. Borghesi et al., Phys. Rev. Lett. 94, 195003 (2005).

[12] M. Roth et al., Phys. Rev. Lett. 86, 436 (2001).

[13] M. Carrié et al., Phys. Plasmas 17, 122707 (2010).

[14] P. K. Patel et al., Phys. Rev. Lett. 91, 125004 (2003).

[15] M. Kaluza et al., Phys. Rev. Lett. 93, 045003 (2004).

[16] A. Flacco et al., Phys. Rev. E 81, 036405 (2010).

[17] E. L. Clark et al., Phys. Rev. Lett. 85, 1654 (2000).

[18] P. Mora, Phys. Rev. Lett. 90, 185002 (2003).

[19] S. C. Wilks et al., Phys. Plasmas 8, 542 (2001).

[20] F. Brunel, Phys. Rev. Lett. 59, 52 (1987).

[21] X. Chen et al., Laser Phys. 21, 198 (2010).

[22] A. Borot et al., Opt. Lett. 36, 1461 (2011).

[23] A. Fukumi et al., Phys. Plasmas 12, 100701 (2005).

[24] G. Bonnaud et al., Laser Part. Beams 9, 339 (1991).

[25] A. Bourdier, Phys. Fluids 26, 1804 (1983).

[26] E. Lefebvre et al., Nucl. Fusion 43, 629 (2003).

[27] S. Fritzler et al., Appl. Phys. Lett. 83, 3039 (2003).

[28] E. Lefebvre et al., J. Appl. Phys. 100, 113308 (2006). 